\title{
Error Analysis of Hit Model under High Mobility
}

\author{
LIU Jun-bang ${ }^{1, a}$, CHEN Yuan-jiang ${ }^{1}$, WU Di-min ${ }^{1}$, JIANG Wei $^{1}$, ZHANG Hua $^{2}$ \\ ${ }^{1}$ Wuhan Mechanical Technology College, Wuhan, China \\ ${ }^{2}$ Wuhan Military Administration, Xiangyang, China \\ aEmail: liujunbang1980@163.com
}

Keywords: Artillery; High-velocity; Hit Model; Firing Lead Error; Hit Probability

\begin{abstract}
The operational effectiveness of the artillery declines under the condition that the artillery and the target are both in motion. The error simulation model is built on the hit model error analysis. In addition, firing lead error and hit probability of the artillery under different operational conditions are calculated with the error simulation model. Influences on hit model error are proposed on the basis of the simulation results. Moreover, measures to improve operational effectiveness in actual combat are proposed.
\end{abstract}

\section{Introduction}

Modern warfare is transforming from mechanization to informationization, and future operational conditions will be significant changed. Both sides in high speed motion at the same time will be the main mode of modern wars. As ground assault forces, the assault gun also faces the above problem.

To meet the operational condition changes, the system performance of assault gun is greatly improved after several upgrade. But it cannot fulfill the high mobility warfare requirements because of the outdated firing theory and implementation techniques utilized in its fire control system. As the mobility of two warring sides significantly enhances, the following factors has become the key factors which restrict the effectiveness of fire control system: (1) Error introduced by simplification of hit model; (2) Shooting delay; (3) High speed motion; (4) Control precision and dynamic process indicators of the gun control system in motion [1].

Among those factors, the first one, i.e. the solving hit model error, is the main factor which influences the fire control system performance under the condition that both the assault gun and the target are in motion. To maximize the operational effectiveness of the existing equipment, this paper intends to analyze the solving hit model error, build the error simulation model, and provide measures to improve operational effectiveness in the actual combat.

\section{Hit Model}

Hit model is one of the basic models of fire control system, and its essence is to determine the coordinates of the projectile and the target track intersection, or the firing lead point, which vary with target motion.

The artillery positions coordinates OXYZ is proposed for convenience, as shown in Fig. 1. Its origin $\mathrm{O}$ is the assault gun position while launching; $\mathrm{M}$ is the target position at the same time, $\mathrm{m}$ is the projection of $\mathrm{M}$ in horizontal, $\mathrm{OX}$ is consistent with Om, OY lies in horizontal and perpendicular to OX. D is the initial slant distance, $\varepsilon$ is the initial elevation angle, $\beta$ is the initial azimuth. $\mathrm{M}_{\mathrm{qv}}$ is the virtual hit point, $\mathrm{m}_{\mathrm{qv}}$ is the projection of $\mathrm{M}_{\mathrm{qv}}$ in horizontal, $\mathrm{D}_{\mathrm{qv}}$ is the slant distance of the virtual hit point, $\varepsilon_{\mathrm{qv}}$ is the elevation angle of the vitual hit point, $\beta_{\mathrm{qv}}$ is the azimuth of the virtual hit point. $\mathrm{M}_{\mathrm{q}}$ is the hit point. 


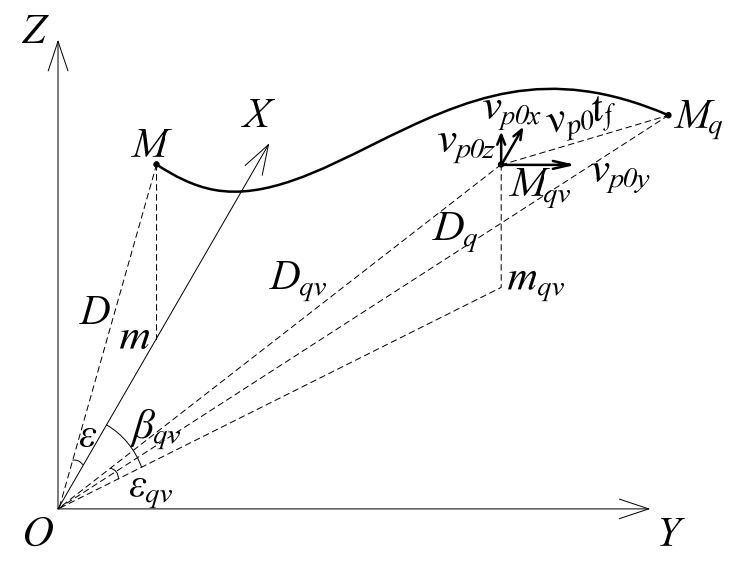

Figure 1. Sketch Map of artillery and target position

Define that velocity of assault gun is $\mathrm{v}_{\mathrm{p} 0}$, and velocity of projectile is $\mathrm{v}_{\mathrm{m}}$, the flight time of projectile is $t_{\mathrm{f}}$, then we have

$$
\left\{\begin{array}{l}
x_{q v}=x_{q}-v_{p 0 x} t_{f}=x_{0}+\int_{0}^{t_{f}} v_{m x}(t) d t-v_{p 0 x} t_{f} \\
y_{q v}=y_{q}-v_{p 0 y} t_{f}=y_{0}+\int_{0}^{t_{f}} v_{m y}(t) d t-v_{p 0 y} t_{f} \\
z_{q v}=z_{q}-v_{p 0 z} t_{f}=z_{0}+\int_{0}^{t_{f}} v_{m z}(t) d t-v_{p 0 z} t_{f} \\
D_{q v}=\sqrt{x_{q v}^{2}+y_{q v}^{2}+z_{q v}^{2}} \\
t_{f}=f\left(D_{q v}, z_{q v}\right)
\end{array}\right.
$$

where $\mathrm{x}_{0}, \mathrm{y}_{0}, \mathrm{z}_{0}$ are the target initial coordinates; $\mathrm{x}_{\mathrm{q}}, \mathrm{y}_{\mathrm{q}}, \mathrm{z}_{\mathrm{q}}$ are the hit point coordinates; $\mathrm{x}_{\mathrm{qv}}, \mathrm{y}_{\mathrm{qv}}, \mathrm{z}_{\mathrm{qv}}$ are the virtual hit point coordinates.

The flight time function $f_{n}(D)$ can be obtained by checking the firing table. When the gun-target attitude angular and shot distance are small, $\mathrm{t}_{\mathrm{f}}$ in Eq. 1 can be calculated by

$$
t_{f}=f\left(D_{q v}, z_{q v}\right) \approx f_{n}\left(D_{q v}\right)
$$

If target is in motion, firing lead is necessary to hit it. Firing lead can be devided into orientational firing lead, i.e. $\Delta \beta=\beta_{q v}-\beta$, and attitude firing lead, i.e. $\Delta \varepsilon=\varepsilon_{q v}-\varepsilon$, which can be formulated as follows:

$$
\left\{\begin{array}{l}
\Delta \beta=\operatorname{arctg}\left(\frac{y_{q v}}{x_{q v}}\right)-\operatorname{arctg}\left(\frac{y_{0}}{x_{0}}\right) \\
\Delta \varepsilon=\operatorname{arctg}\left(\frac{z_{q v}}{\sqrt{x_{q v}^{2}+y_{q v}^{2}}}\right)-\operatorname{arctg}\left(\frac{z_{0}}{\sqrt{x_{0}^{2}+y_{0}^{2}}}\right)
\end{array}\right.
$$

\section{Hit Model Error under High Mobility}

\section{Hit Model Error Analysis}

Hit model is simplified in fire control system realization due to the limitation of engineering, battlefield environment and other factors. Firing lead is calculated by [2]

$$
\Delta \beta=\omega_{\beta} t_{f}, \Delta \varepsilon=\omega_{\varepsilon} t_{f}
$$

where $\omega_{\beta}$ and $\omega_{\varepsilon}$ is the relative horizontal and vertical angular velocity of target.

From Eq. (4) we know that, fire control system is designed to accurately predict firing lead by using the flight time of the projectile and the average relative angular velocity between the target and the artillery. And these two quantities exist prediction error.

As shown in Fig. 2, at first the target is at the point $\mathrm{M}_{0}$, the distance obtained by laser ranging is 
$\mathrm{D}_{0}$. The artillery fires after the system response time $\mathrm{T}_{\mathrm{s}}$ when the target move to point $\mathrm{M}_{\mathrm{f}}$. The target arrives at the meeting point $\mathrm{M}_{\mathrm{q}}$ within the projectile flight time $\mathrm{t}_{\mathrm{f}}$. The virtual meeting point is $\mathrm{M}_{\mathrm{qv}}$. Fire control system uses $\mathrm{D}_{0}$ to predict projectile flight time $t_{\mathrm{fc}}$, and the projectile's real flight time $t_{\mathrm{f}}$ and $\mathrm{D}_{\mathrm{qv}}$ also conform to the flight time function. Therefore, the flight time estimation error is:

$$
\Delta t_{f}=t_{f_{c}}-t_{f}=f_{n}\left(D_{0}\right)-f_{n}\left(D_{q v}\right)
$$

Fire control system estimates the relative angular velocity of the target by using simpling in two seconds before firing [3]. The average relative angular velocity error can be written as

$$
\left\{\begin{array}{l}
\Delta \overline{\omega_{\beta}}=\bar{\omega}_{\beta}-\bar{\omega}_{\beta f}=\frac{1}{8} \sum_{i=0}^{8} \omega_{\beta i}-t_{f}^{-1} \int_{0}^{t_{f}} \omega_{\beta f}(t) d t \\
\Delta \overline{\omega_{\varepsilon}}==\bar{\omega}_{\varepsilon}-\bar{\omega}_{\varepsilon f}=\frac{1}{8} \sum_{i=0}^{8} \omega_{\varepsilon i}-t_{f}^{-1} \int_{0}^{t_{f}} \omega_{\varepsilon f}(t) d t
\end{array}\right.
$$

where $\Delta \omega_{\beta}, \Delta \omega_{\varepsilon}$ are the relative average angular velocity errors, $\omega_{\beta \mathrm{i}}, \omega_{\varepsilon \mathrm{i}}$ are the sampling used by fire control system, $\omega_{\beta f}, \omega_{\varepsilon f}$ are the relative angular velocity of the target with respect to the gun firing positoin during the projectile flight time.

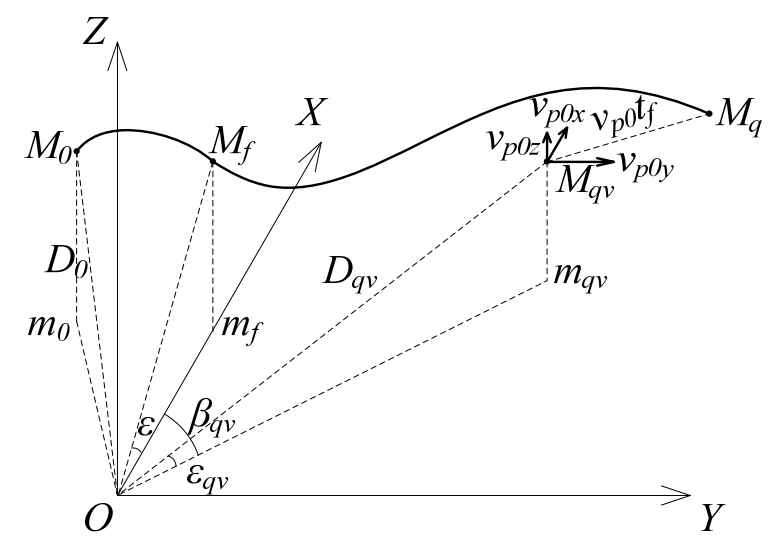

Figure 2. Sketch Map of error

\section{Error Simulation Model}

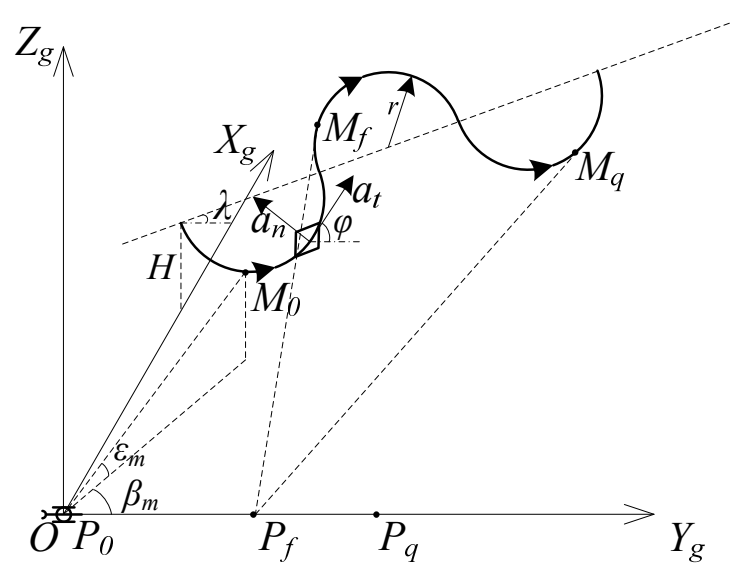

Figure 3. Motion of artillery and target

The trajectories of the artillery and the target can be divided into lines and curves. Acceleration mode can be divided into uniform (or stationary), uniform acceleration, and variable acceleration. The instant positonal relationship of the artillery and the target in the shooting process is shown in Fig. 3.

In Fig. 3, $\mathrm{OX}_{\mathrm{g}} \mathrm{Y}_{\mathrm{g}} \mathrm{Z}_{\mathrm{g}}$ is the ground coordinate. Its origin $\mathrm{O}$ is the initial position of artillery. $\mathrm{OY}_{\mathrm{g}}$ is consistent with artillery movement, $\mathrm{OXgYg}$ is parallel with the horizontal plane; $\mathrm{M}_{0}, \mathrm{P}_{0}$ represent the position of target and artillery at the ranging moment, respectively; $\mathrm{M}_{\mathrm{f}}, \mathrm{P}_{\mathrm{f}}$ represent the position of target and artillery at the launching moment, respectively; $\mathrm{M}_{\mathrm{q}}, \mathrm{P}_{\mathrm{q}}$ represent the position of target and artillery at the hit moment, respectively. $\varphi$ is the azimuth angle of target; $\lambda$ is the route angle of target $\mathrm{S}$-shaped maneuver; $r$ is the turning radius of target $S$-shaped maneuver; $a_{\operatorname{tg}}$ is the tangential acceleration; $\mathrm{a}_{\mathrm{ng}}$ is the normal acceleration; $\mathrm{H}$ is the elevation difference between artillery and target; $\beta_{\mathrm{m}}, \varepsilon_{\mathrm{m}}$ are azimuth angle and elevation angle of the line cross artillery and target, respectively.

The motion of target can be fomulated as follows,

$$
\left[\begin{array}{l}
v_{x g} \\
a_{x g} \\
v_{y g} \\
a_{y g}
\end{array}\right]=\left[\begin{array}{llll}
0 & 1 & 0 & 0 \\
0 & 0 & 0 & 0 \\
0 & 0 & 0 & 1 \\
0 & 0 & 0 & 0
\end{array}\right]\left[\begin{array}{l}
x_{g} \\
v_{x g} \\
y_{g} \\
v_{y g}
\end{array}\right]+\left[\begin{array}{cc}
0 & 0 \\
\sin \varphi & \cos \varphi \\
0 & 0 \\
\cos \varphi & \sin \varphi
\end{array}\right]\left[\begin{array}{l}
a_{t g} \\
a_{n g}
\end{array}\right]
$$

where $\mathrm{x}_{\mathrm{g}}, \mathrm{y}_{\mathrm{g}}$ represent target position; $\mathrm{v}_{\mathrm{xg}}, \mathrm{v}_{\mathrm{yg}}$ represent target velocity; $\mathrm{a}_{\mathrm{xg}}, \mathrm{a}_{\mathrm{yg}}$ represent target acceleration.

And the relative angular velocity of target can be calculated by

$$
\left[\begin{array}{l}
w_{\beta} \\
\omega_{\varepsilon}
\end{array}\right]=\left[\begin{array}{l}
v_{r r} / D \\
v_{d r} / D
\end{array}\right]
$$


where

$$
\left[\begin{array}{l}
v_{r r} \\
v_{e r} \\
v_{d r}
\end{array}\right]=\left[\begin{array}{l}
v_{x g}-v_{p x g} \\
v_{y g}-v_{p y g} \\
v_{z g}-v_{p z g}
\end{array}\right] \cdot\left[\begin{array}{ccc}
-\cos \beta_{m} & \sin \beta_{m} & 0 \\
\sin \beta_{m} \cos \varepsilon_{m} & \cos \beta_{m} \cos \varepsilon_{m} & \sin \varepsilon_{m} \\
-\sin \beta_{m} \sin \varepsilon_{m} & -\cos \beta_{m} \sin \varepsilon_{m} & \cos \varepsilon_{m}
\end{array}\right]
$$

is the relative velocity in artillery sight coordinate,

$$
D=\sqrt{\left(x_{g}-x_{p g}\right)^{2}+\left(y_{g}-y_{p g}\right)^{2}+\left(z_{g}-z_{p g}\right)^{2}}
$$

is the shot distance.

Therefore, the firing lead can be obtained

$$
\Delta \beta_{c}=\bar{\omega}_{\beta} \bullet f_{n}\left(D_{0}\right), \Delta \varepsilon_{c}=\bar{\omega}_{\varepsilon} \bullet f_{n}\left(D_{0}\right)
$$

\section{Simulation and Analysis}

Suppose that the altitude difference of artillery and target is less than $200 \mathrm{~m}$, the motion of artillery is uniform linear while shooting, and the velocity range is $5 \sim 20 \mathrm{~km} / \mathrm{h}$. The radius range of S-shaped maneuver of target is $10 \sim 200 \mathrm{~m}$, and the velocity range is $5 \sim 72 \mathrm{~km} / \mathrm{h}$, the acceleration range is $0 \sim 3.0 \mathrm{~m} / \mathrm{s}^{2}$, the heading angle range is $90^{\circ} \sim-90^{\circ}$. The shot distance is set to be $2000 \mathrm{~m}$.

\section{Uniform Linear Motion}

The target velocity step is $2 \mathrm{~m} / \mathrm{s}$. The maximums of horizontal and vertical firing lead error are 0.015 mil and 0.006 mil, respectively. The hit probability curve is shown in Fig. $4\left(a_{t}=0.0 \mathrm{~m} / \mathrm{s}^{2}\right)$. It can be seen that hit probability varies with heading angle and target velocity among $52.5 \% \pm 2.0 \%$. And hit probability of opposite movement is higher than that of contrary motion.

\section{Uniform Acceleration Linear Motion}

The target velocity step is $2 \mathrm{~m} / \mathrm{s}$. And the target acceleration step is $0.5 \mathrm{~m} / \mathrm{s}^{2}$. The results are shown in Figs. 5 and 6. It is seen that the error increases significantly as acceleration increases. The maximum horizontal and vertical firing lead errors are 2.74 mil and 0.14 mil, respectively. As shown in Figure 8, hit probability varies with route angle and initial velocity in the range of $44.8 \%$ $\sim 54.6 \%$. When route angle is $0^{\circ}$, acceleration of target increases from 0 to $2.5 \mathrm{~m} / \mathrm{s}^{2}$, hit probability decreases from $52.6 \%$ to $45.0 \%$.

\section{Variable Acceleration Linear Motion}

The variation of acceleration [5] is given in Fig. 7. The target initial velocity step is set to $2 \mathrm{~m} / \mathrm{s}$. The maximum horizontal and vertical firing lead errors are $0.92 \mathrm{mil}$ and $0.046 \mathrm{mil}$, respectively. Hit probability varies with route angle and target in range of $51.0 \% \sim 54.4 \%$. The hit probability of opposite motion is higher than that of contrary motion. Hit probability increases slightly it the target velocity declines in shooting process..

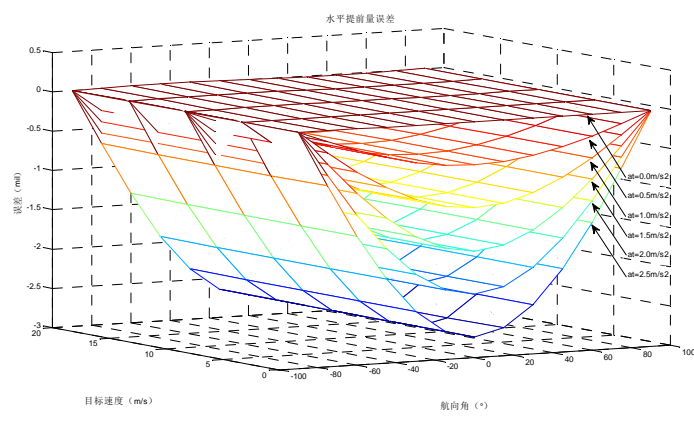

Figure 4. Horizontal firing lead error under uniform acceleration motion

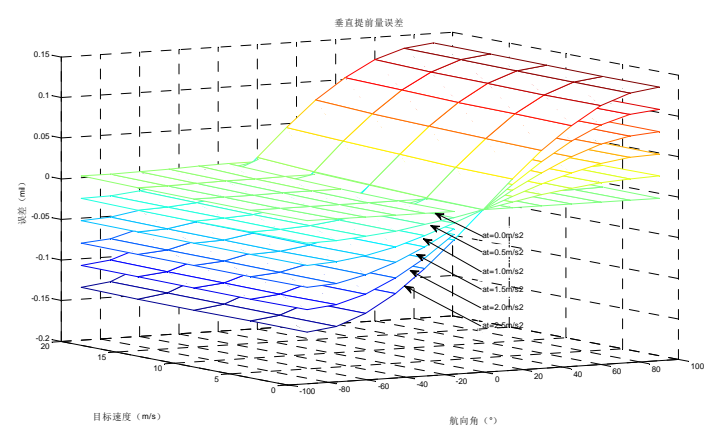

Figure 5. Vertical firing lead error under uniform acceleration motion 


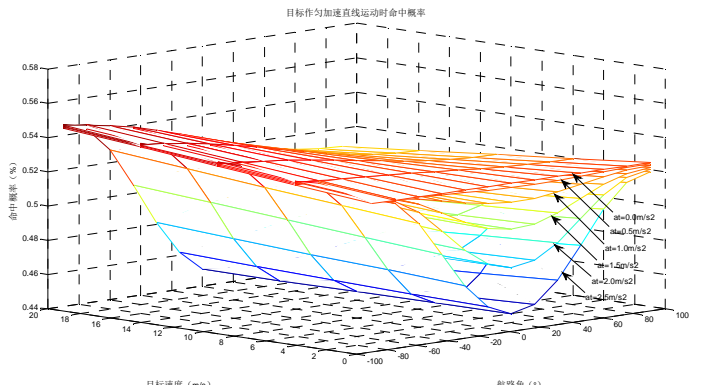

Figure 6. Hit probability under uniform acceleration motion

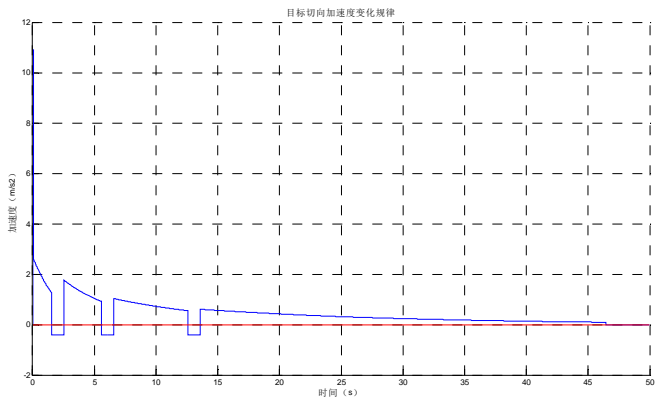

Figure 7. Variation of acceleration

\section{Uniform Acceleration S-shaped Maneuver}

Let $\mathrm{a}_{\mathrm{t}}=2.5 \mathrm{~m} / \mathrm{s}^{2}, \mathrm{v}_{0}=5 \mathrm{~m} / \mathrm{s}$. The target turning radius step is set to $1 \mathrm{~m}$. The results are shown in Figs. 8 and 9. It is seen that the maximum horizontal and vertical firing lead error are 21.26 mil and 1.08 mil, respectively. As shown in Fig. 10, hit probability varies with turning radius, route angle and initial velocity within $9.1 \% \sim 53.9 \%$ range. The influence of acceleration can be ignored. Hit probability is high when radius is less than $20 \mathrm{~m}$ and route angle is $0^{\circ}$. Route angle which has the highest hit probability increases from $0^{\circ}$ to $90^{\circ}$ as radius raises.

The variation of firing lead error and hit probability under variable acceleration S-shaped maneuver is similar to that under uniform one..

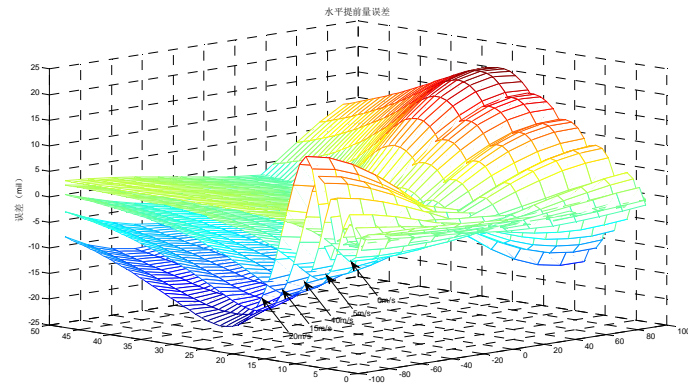

Figure 8. Horizontal firing lead error under uniform acceleration S-shaped maneuver

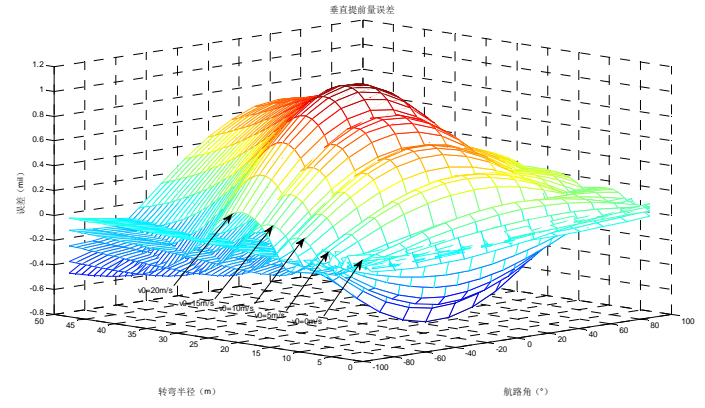

Figure 9. Vertical firing lead error under uniform acceleration S-shaped maneuver
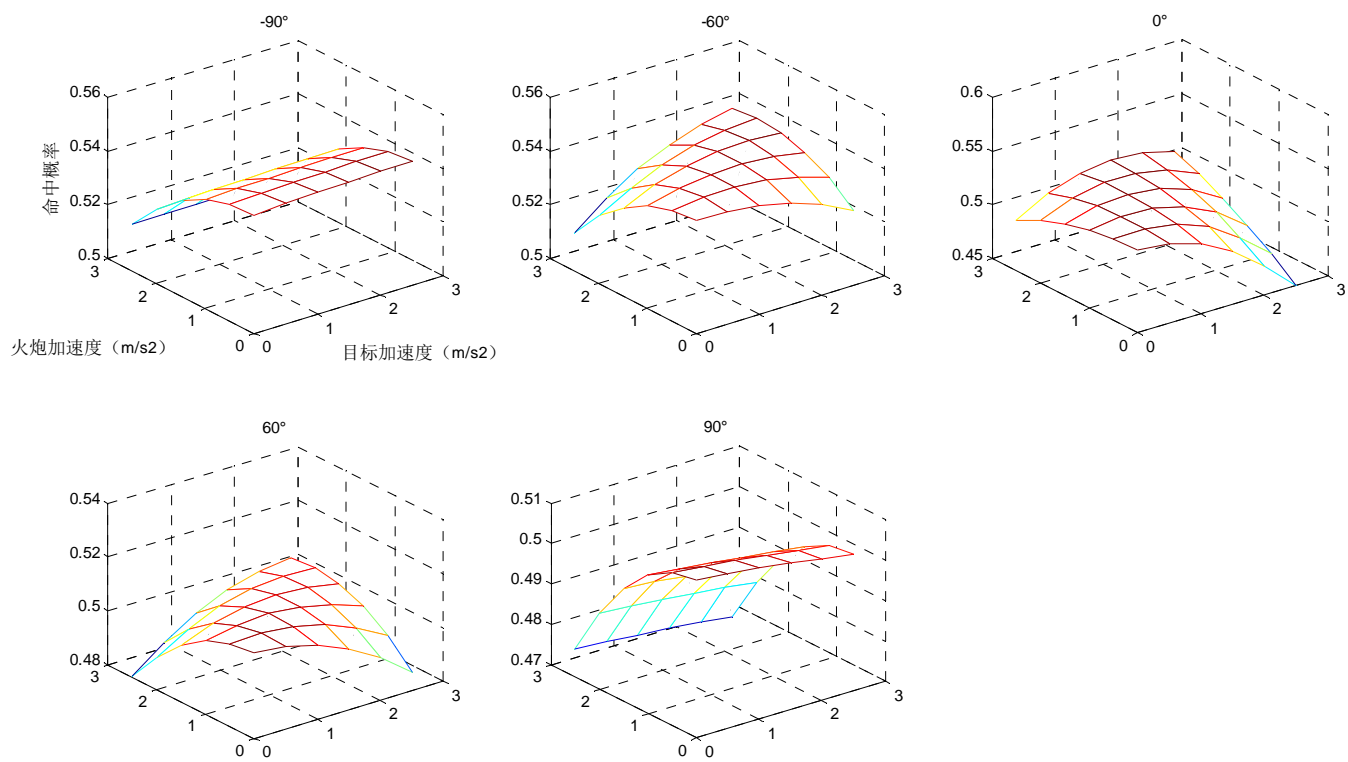

Figure 10. Hit probability when artillery and target in different route angle 


\section{Conclusion}

By comparison of effects under different target motions, firing lead error of shooting against the S-shaped maneuvering target is significantly higher than those of shooting against other motions. And hit probability is the least of all. For non-S-shaped maneuver target, hit probability is the highest when route angle is $90^{\circ}$. For S-shaped maneuver target, hit probability is the highest when turning radius is less than $20 \mathrm{~m}$ and route angle is $0^{\circ}$. Route angle which has the highest hit probability transits to $-90^{\circ}$ as the turning radius increases.

When route angle of target is $\pm 90^{\circ}$, shot in still or uniform motion will get higher hit probability. For other conditions, hit probability is higher when accelerations of artillery and target are close.

\section{References}

[1] Wang Qinzhao, et al. Tank fire control system based on high mobility conditions [J], Ordnance Industry Automation, 2012, 31 (3): 19-21.

[2] Zhou Qihuang. Digital Tank Fire Control System Fundamentals [M]. Beijing: Weapon Industry Press, 1991.

[3] Wang Qinli, et al. Fire Control System Error stabilization study [J], Gun Launch \& Control, 2003 (S1): 16-18.

[4] Shou Shaojun. Tank sighting system coordinate system [J], Fire Control \& Command Control, 2006 (5): 37-40.

[5] Li Guanghui, et al. Mobility M1A2 tank system simulation [J], Computer simulation, 2001 (6): 4-6.

[6] General Staff. $x \times \times$ wheeled artillery on their own weapons and weapons systems operating tutorial [M], Beijing: PLA Publishing House, 2004. 